\title{
Integrating Kinect Depth Data with a Stochastic Object Classification Framework for Forestry Robots
}

\author{
Mostafa Pordel, Thomas Hellström and Ahmad Ostovar \\ Umeå University, Umeå, Sweden
}

\begin{abstract}
Keywords: Image Processing, Depth Integration.
Abstract: In this paper we study the integration of a depth sensor and an RGB camera for a stochastic classification system for forestry robots. The images are classified as bush, tree, stone and human and are expected to come from a robot working in forest environment. A set of features is extracted from labeled images to train a number of stochastic classifiers. The outputs of the classifiers are then combined in a meta-classifier to produce the final result. The results show that using depth information in addition to the RGB results in higher classification performance.
\end{abstract}

\section{INTRODUCTION}

The major contribution of this paper is a study of the effects of adding depth sensor information to an object classification system that uses only RGB camera. The system is aimed for a forestry robot and the task is to classify images as one of the four classes bush, tree, stone and human. In this paper object classification is used to make the forestry robot capable of conducting applied tasks such as navigating in forest environments, collecting information about interest objects etc. Object classification for this kind of Resource Constrained Embedded Systems (RCES) is an emerging research area where real-time performance is one of the main criteria for developed algorithms e.g. realtime vision system for simultaneous localization and mapping(Davison et al., 2007). In this work all the needed calculations are done in less than a second that is fast enough for the experimental level for RCESs. Also it is possible to improve the calculation time by using more powerful operating systems. In general, object classification methods are either bottomup or top-down (Kokkinos et al., 2006) (Fang et al., 2011). Top-down methods typically include matching input data to a certain model or features of objects (see e.g. (Xiao et al., 2010)). Bottom-up approaches start with low level features of the image, like depthbased features, color-based features etc. (see e.g. (He et al., 2011)). More specifically, stochastic classifiers in bottom-up style are studied for integration of depth and RGB sensor features. A set of all features including depth and RGB are used to train classifiers in a three tier architecture for object detection as illustrated in Figure 1. It is possible to add very sophisticated edge and corner features such that the four objects (trees, bushes, stones and humans) would be detected easier. However this is beyond the main goal of this paper, which is to study the integration of depth sensor information in a generic stochastic classification method. Finally, depth information is used to facilitate image labeling.

We extract 12 color-based features from RGB images, inspired by a project (Ästrand and Baerveldt, 2002) with classification of sugar beets and weeds for agricultural robots. Depth images are used to calculate additional 6 features. The system uses five stochastic classifiers and a Weighted Majority Vote (WMV) method as a meta-classifier (Robert et al., 2003) to fuse the individual classifiers. Classification results are presented for three cases; color-based features extracted from the entire image, color-based features extracted from the color foreground image and finally color-based and depth-based features extracted from the color foreground image and depth image. A database of images has been collected and labeled with a tool described in Section 2. Section 3 describes how the different components of the system are connected. The sensor device is presented in Section4. The used features are described in Section 5. Section 6 explains the used classifiers and also how these classifiers are combined together to increase the overall performance. The results are presented in Section 7 and the effect of adding the depth sensor is analyzed. Finally, conclusions and future research are described 


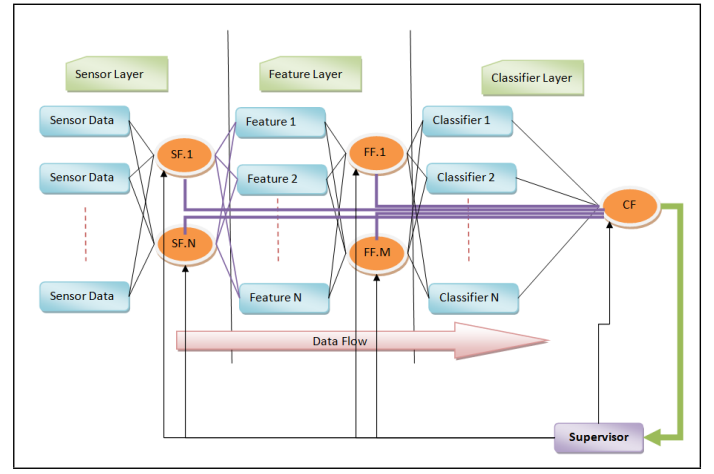

Figure 1: Framework for generic stochastic feature-based object classification. (SF: Sensor Fusion, FF: Feature Fusion, CF: Classifier Fusion).

in Section 8.

\section{ODAR TOOL}

ODAR (Object Detection for Agriculture Robots) is a tool that integrates software components written in C\#, MATLAB and $\mathrm{C}++$ for image analysis (see Figure 2). It interacts with sensors and collects images into a database. ODAR also contains functionality for image labeling. The user may select the desired object in an RGB image and associate it with a specific class. This process is called labeling. More specifically, ODAR uses an algorithm that selects an area when the user clicks on the image. It uses a filter to search for connected pixels with similar RGB values. For objects with many different colors e.g. a human with clothes with many colors, the user may click on multiple points to cover the entire object. This interactive solution works well, but the procedure is time consuming and requires multiple clicks for some images. For this reason, we developed an automatic method that uses depth images for object selection within the RGB image, by removing the background pixels from the $R G B$ image. The procedure is similar to what is described in Section 4.2 for background detection used for calculation of features. In our experiment, all images have been labeled with this method.

\section{SYSTEM ARCHITECTURE}

One way to increase the performance of an existing classification solution is to add and fuse new components, e.g. new sensors, features or classifiers. Our system is built on a framework (Figure 1) that supports this approach. It comprises a Sensor Layer, a

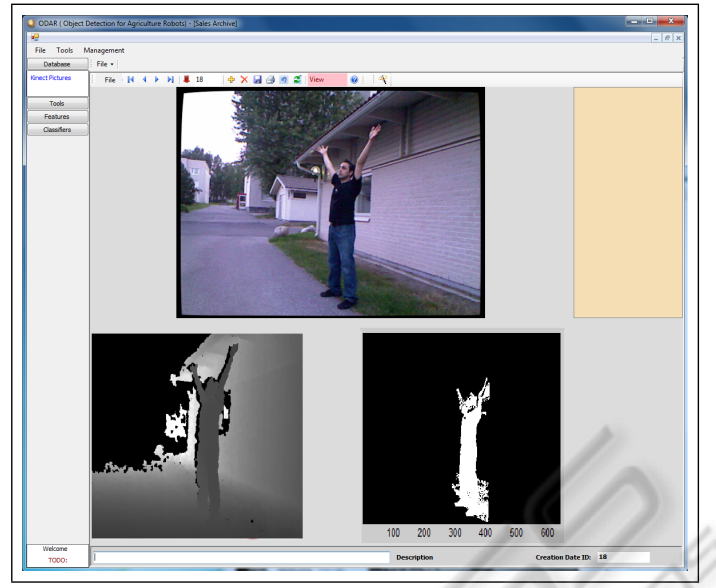

Figure 2: ODAR is a tool that supports image analysis. It collects images to a database and computes foreground images by using RGB and depth images.

Feature Layer and a Classification Layer. In each layer, fusion of information may take place. In this paper, a depth sensor and an RGB camera are fused in the Sensor Layer to generate foreground images. In the Feature Layer, 18 different depth-based and RGBbased features are generated as described in Section 5. Finally, in the classification layer, classifier outputs are fused to generate a combined output. In the following, the three layers will be described in more detail.

\section{SENSOR LAYER}

This layer reads and fuses data from sensors (or from the ODAR database) and provides the inputs for feature extraction in the Feature layer. In the remainder of this section of paper, the Kinect sensor and sensor fusion for background detection are presented.

\subsection{Microsoft Kinect}

In late 2010 Microsoft released the Kinect Device for the Xbox console. Since then, several groups within the robotics community have integrated Kinect with their applications. ROS (Robotic Operating System) (Van den Bergh et al., 2011) supports the device, which increased its usefulness in robotics. In summer 2011, Microsoft introduced the SDK for Kinect with a user friendly API that makes interaction much easier. Kinect has an RGB camera, an infrared based depth camera and a row of microphones. In this research the microphones are not used. The depth camera works well indoors in the limited range of 0.4 to 9 meters but has poor performance outdoors, in partic- 
ular in sharp sunlight. However, we have successfully used the Kinect camera for the experiments reported in this paper. To collect a proper set of images we concerned some limitations such as distance of object to the camera, the strength and direction of sunlight. The developed solutions can of course also be used with other kinds of sensors that provide depth information e.g. laser scanner which has wider working range and higher depth image quality in different lightning conditions in comparison to Kinect depth sensor.

\subsection{Background Detection}

The object of interest (the one that is the basis for the labeling) in each picture is likely to be located in the foreground of image. Background detection makes it possible to compute features for the interesting object, rather than for the entire image. Background detection is often discussed and solved for sequences of images or video frames rather than single RGB images. One common main technique in video background detection uses the differences between consecutive video frames to detect the background (Cucchiara et al., 2003). However, this approach is not applicable for single images or for videos where the interesting objects are not moving, such as stones in forest environments. Another common technique, which is applicable for single images, uses morphological filters to classify a specific pattern as background (e.g. Poor lighting in parts of image) (Jimenez-Sanchez et al., 2009). This approach is challenging in forest environments where objects and backgrounds are very similar. Another possibility is to add a range sensor like time of flight (TOF) sensor (Van den Bergh and Van Gool, 2011) or stereo vision and build a 3D model and detect the background using depth information. In this project, we use a simple background detection algorithm based on depth information and the fact that is very likely in our object classification problem that the object of interest would be located in foreground image. The background mask $B(I, j)$ is computed from a depth image $D(i, j)$, as follows:

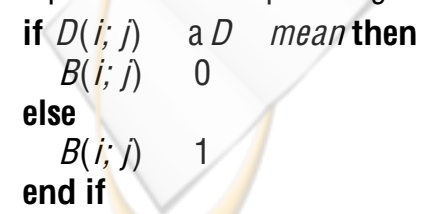

Where $D$-mean is the mean value of all elements in $D$. This calculation is repeated for all pixels $(i, j)$ in $D$. The depth foreground image is then computed by multiplying $D$ element wise with mask $B$. For the Kinect camera, $D$ has lower resolution than RGB image. $B$ is therefore resized and then multiplied element wise with the $R G B$ image to generate the $R G B$

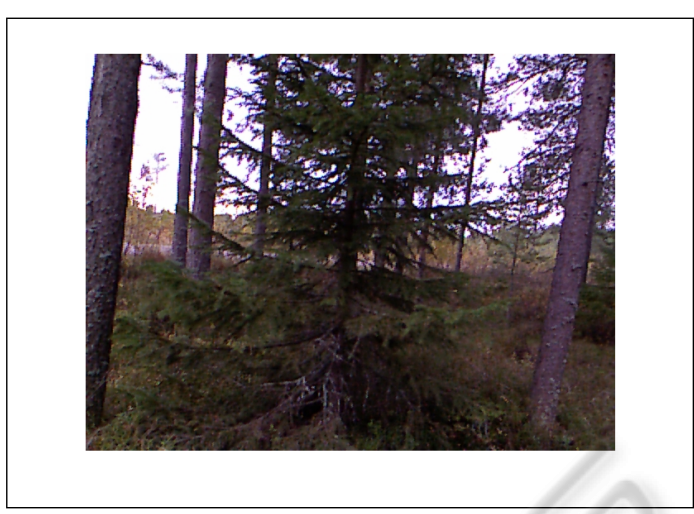

Figure 3: RGB Image of a tree.

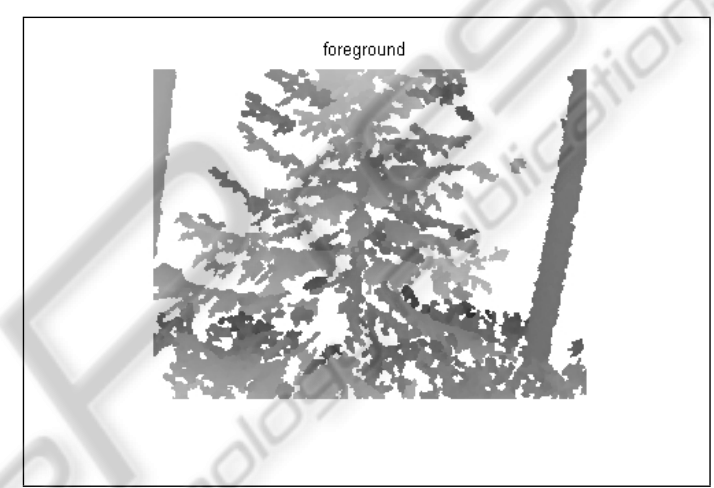

Figure 4: Depth values from the Kinect camera is used to detect the foreground objects of Figure 3.

foreground image. Parameter a is a number between 0 and 1 and determines how far from $D$-mean pixels are considered to be in foreground image rather than background. It is set with maximum likelihood estimation using a part of the training images, such that manually marked foregrounds match the computed foregrounds the most. For the images in our database, $\mathrm{a}=0.5$ was computed as optimal.

The computation of depth foreground and color foreground images is an example of how sensor data may be fused to produce new sensor data that can be used by the subsequent Feature layer. Figure 4 shows how the objects of interest appear in the Depth foreground image. All depth-based features are extracted from Depth foreground images while color-based features are calculated from RGB foreground images (in the result section, results with color-based features calculated from the entire RGB images are also reported). 


\section{FEATURE LAYER}

In total, 18 features are extracted for each image pair (RGB and Depth). They represent either color-based or depth-based properties. Depth-based features are extracted from depth foreground images (see Section 4.2). Hue, Saturation and Value (HSV) images are calculated from the RGB images and are less sensitive to image intensity and vary less to varying light conditions. Therefore they often lead to better performance in classification problems (Chen et al., 2007). For the Hue, Saturation and Value images, as well as for each color channel (R, G and $B$ ) the mean and variance are computed as separate features. If the generated features were directly given to the classifiers, features with larger ranges would dominate smaller features effects. Therefore, all features are normalized to zero mean and unity standard deviation.

All color-based features are listed in Table 1.

Table 1: Color-based Features.

\begin{tabular}{|l|l|}
\hline R,G,B-Mean & Mean values of red, green, blue \\
\hline R,G,B-Var & Variances of red, green, blue \\
\hline H,S,V-Mean & Mean values of Hue, Saturation, Value \\
\hline H,S,V-Var & Variances of Hue, Saturation, Value \\
\hline
\end{tabular}

Table 2 shows depth-based features that are extracted from depth foreground images. The Skeleton feature is based on the idea of removing maximum number of pixels in the image without losing the overall connectivity in the image. The value of the Skeleton feature equals the number of remaining pixels. This feature has been used for a long time in computer vision, optical character recognition and pattern classification (Lakshmi and Punithavalli, 2009). The Skeleton feature in Figure 6 is computed for the depth foreground image in Figure 4.

Table 2: Depth-based features.

\begin{tabular}{|l|l|}
\hline D-mean & Mean value of foreground depth image \\
\hline D-var & Variance of foreground depth image \\
\hline Skeleton & Number of pixels in Skeleton image \\
\hline Dispersion & Distribution of foreground depth image \\
\hline Perimeter & Length of perimeter of foreground depth image \\
\hline Area & Area of foreground object \\
\hline
\end{tabular}

The Area feature is computed as the number of pixels in the depth foreground image. The Dispersion feature is calculated by dividing the Skeleton feature by the Area. For stones, which typically have low depth variations, the Skeleton feature is small. The Dispersion is also small for stones compared to trees or bushes where, for the same Area, the Skeleton feature is larger. In short, Dispersion shows how distributed an object is over the image. The Dispersion feature in Figure 9 is computed for the depth foreground image in Figure 4.

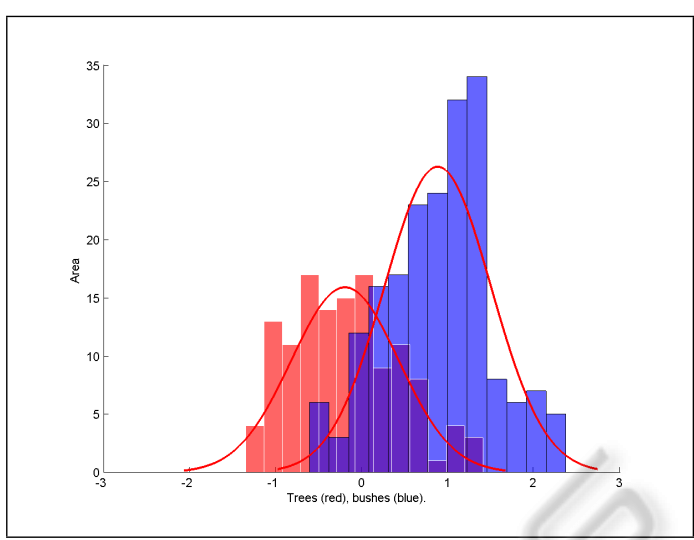

Figure 5: Class histograms and fitted distributions for the Area feature. It helps distinguishing trees from bushes.

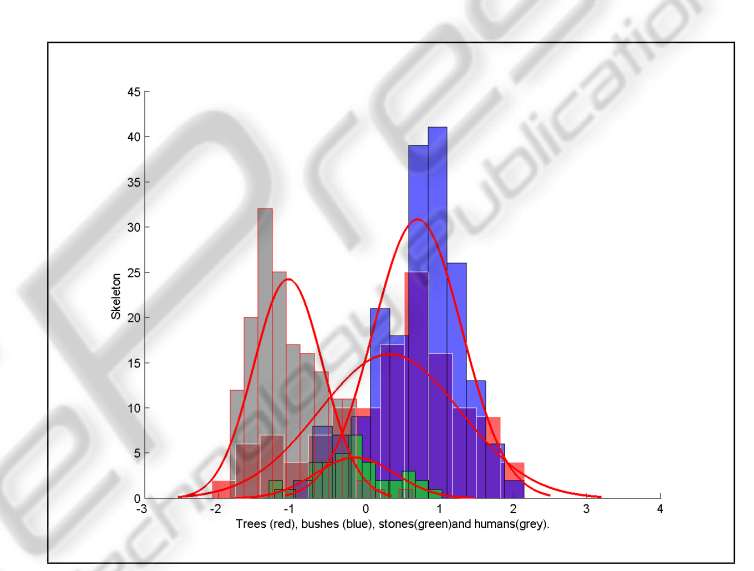

Figure 6: Class histograms and fitted distributions for the Skeleton feature.

The computation of Dispersion feature is an example of how features may be fused to produce additional features for the subsequent Classifier layer.

Depth features help distinguishing trees from bushes, which can be a hard task even for humans. Images with trees usually include trunks that have less variation in depth and also smaller depth mean. Figure 5 illustrates how trunks effect depth information.

Perimeter is another feature that gives information about shape of the object. It is computed as the number of pixels in the identified perimeter in the image (see Figure 7).

Figure 8 shows the distribution of normalized perimeter for the collected images of trees, bushes, stones and human.

\section{CLASSIFIER LAYER}

Five stochastic classifiers are used for classification: Naive Bayes (Friedman et al., 1997), Decision Trees 


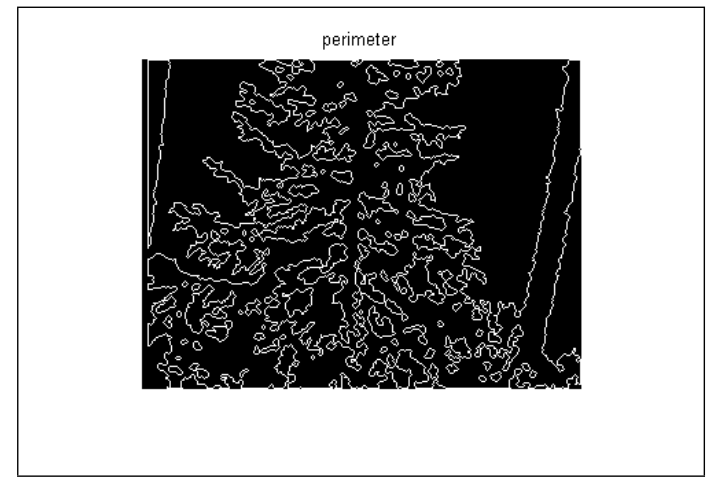

Figure 7: The Perimeter for the depth foreground image shown in Figure 4. The Perimeter feature is the number of pixels in the perimeter.

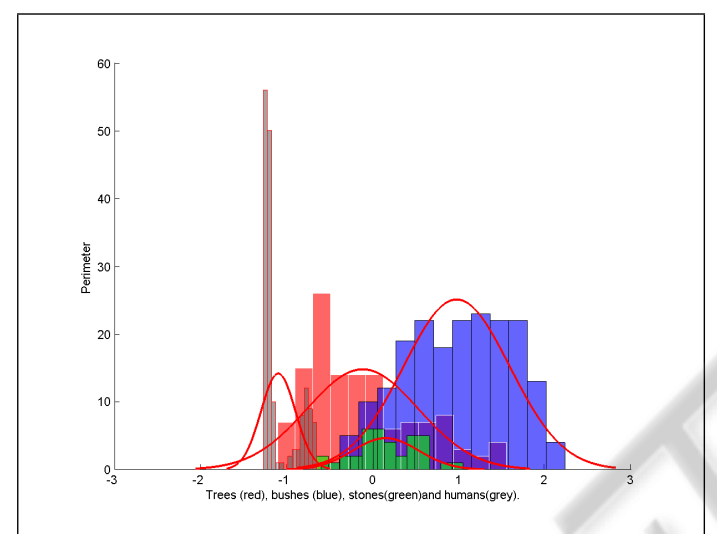

Figure 8: Class histograms and fitted distributions for the Perimeter feature.

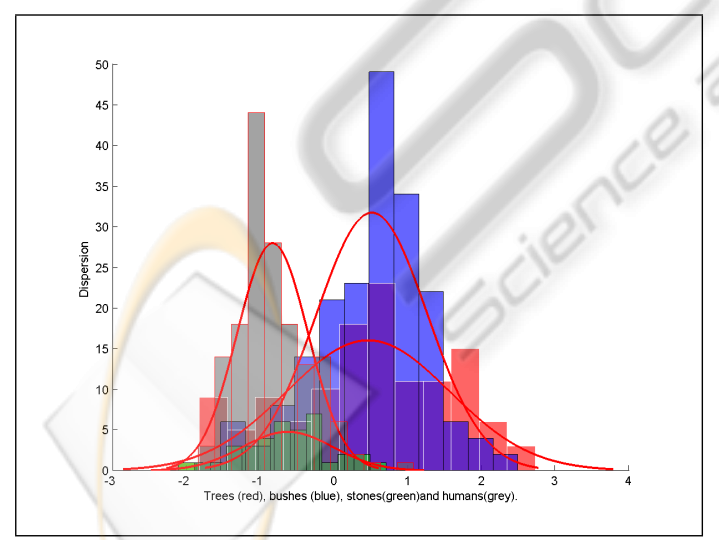

Figure 9: Class histograms and fitted distribution for the normalized Dispersion feature.

(Wang et al., 2008), K-Nearest Neighbor (KNN) (Zhang et al., 2006), Support Vector Machine (SV M) (Li et al., 2009) and Linear Discriminant Analysis (LDA) (An et al., 2009). These classifiers are generating the naive results that are given to a meta-classifier to be fused in order to make the final result. All men- tioned classifiers, except SVM, train very fast. With a 3 gigahertz processor, the entire training phase (excluding SV M) with 650 images, (see Section 2) takes less than one second, which is fast enough also for real-time online training (see (Wang et al., 2008), (An et al., 2009)) if the system would be implemented on a real-time platforms like FPGA (Pordel et al., 2011), (An et al., 2009). SVM trains slower, but all methods including SVM (Li et al., 2009) can provide realtime classification after training. The basic version of an SVM classifier only works with two classes. In this project we use the "One Versus Rest" technique (Zhang et al., 2007) to extend the SVM to work with four classes.

Table 3: Accuracy without background subtraction using color-based features only.

\begin{tabular}{|l|l|l|}
\hline Classifier & $\begin{array}{l}\text { Training } \\
\text { accuracy }\end{array}$ & $\begin{array}{l}\text { Testing } \\
\text { accuracy }\end{array}$ \\
\hline KNN & $94.74 \%$ & $90.51 \%$ \\
\hline DT & $95.84 \%$ & $77.22 \%$ \\
\hline SVM & $93.91 \%$ & $90.51 \%$ \\
\hline NB & $86.15 \%$ & $79.75 \%$ \\
\hline LDA & $96.687 \%$ & $77.85 \%$ \\
\hline WMV & - & $89.87 \%$ \\
\hline
\end{tabular}

All classifier outputs are combined by Weighted Majority Vote rule (WMV)(De Stefano et al., 2002). WMV does not need training, but uses the accuracy of the individual classifiers to determine the weights for each classifier. The accuracy $A(i)$ (fraction correct classifications) for each classifier $i$ is computed by averaging the accuracy from 10 -folds cross validation. $w(i)$, the weight for classifier $i$ is computed such that all weights add up to 1 :

$$
w(I)=A(I)=\left(\AA_{j=1}^{5} A(j)\right)
$$

The WM V classifier computes a fused classification $f$ according to:

$$
f=\underset{i}{\arg \max } \AA_{j=1}^{5}(w(j) \partial /(C(J)=i))
$$

Where $C(j)$ is the classification delivered by classifier $j$ and $I$ is an indicator function (James and Stanford University, 1998) computing to 1 if the argument is true and to 0 otherwise. In other words, $f$ is the class with the largest number of weighted votes.

\section{RESULTS}

As a rule of thumb, the training set should be about 10 times the number of features used in classification (Jain et al., 2000). For the 18 features that are 
Table 4: Accuracy with background subtraction using colorbased features only.

\begin{tabular}{|l|l|l|}
\hline Classifier & $\begin{array}{l}\text { Training } \\
\text { accuracy }\end{array}$ & $\begin{array}{l}\text { Testing } \\
\text { accuracy }\end{array}$ \\
\hline KNN & $93.35 \%$ & $87.34 \%$ \\
\hline DT & $96.12 \%$ & $83.54 \%$ \\
\hline SVM & $91.14 \%$ & $89.24 \%$ \\
\hline NB & $83.93 \%$ & $68.35 \%$ \\
\hline LDA & $97.51 \%$ & $93.67 \%$ \\
\hline WMV & - & $93.04 \%$ \\
\hline
\end{tabular}

Table 5: Accuracy with background subtraction using colorbased and depth-based features.

\begin{tabular}{|l|l|l|}
\hline Classifier & $\begin{array}{l}\text { Training } \\
\text { accuracy }\end{array}$ & $\begin{array}{l}\text { Testing } \\
\text { accuracy }\end{array}$ \\
\hline KNN & $97.23 \%$ & $94.94 \%$ \\
\hline DT & $97.51 \%$ & $81.65 \%$ \\
\hline SVM & $93.91 \%$ & $94.30 \%$ \\
\hline NB & $89.75 \%$ & $72.15 \%$ \\
\hline LDA & $98.06 \%$ & $88.61 \%$ \\
\hline WMV & - & $96.20 \%$ \\
\hline
\end{tabular}

described in Section 5, 150 samples have been collected for each object type and the database has in total $650 \mathrm{RGB}$ and depth images. From this set, $80 \%$ is used for training, $10 \%$ for 10 fold cross-validation of the classifiers and the last $10 \%$ for final testing of WMV meta-classifier. The entire process is repeated 10 times for 10 fold cross-validation also for WM V. In order to study the effect of adding depth information, classification results are computed for three different cases. Classification results for the five classifiers and the fused WMV classifier are presented in Tables 3, 4 , and 5 . Accuracy for both training data and test data are presented and is computed as the fraction of successful classifications over the respective set.

The results for the first case, color-based features extracted from the entire input images (without background subtraction) have the worst performance.

In the second case, with color-based features computed from color foreground images, the classification results are better. The reason is that the features are computed for a more relevant part of the images, and hence are less noisy compared to the first case.

In the third case, depth-based and color-based features are extracted from foreground images. The results show how the added depth-based features improve the classification accuracy.

Table 6, shows the confusion matrix for WM V and case three. Of special interest is the low confusion between trees and bushes, which may be hard to distinguish between also for a human.

\section{CONCLUSIONS AND FUTURE WORKS}

The results obviously show that using depth sensor information for subtracting background and adding depth based features improves the classification accuracy. Background subtraction is essential for computation of all features and can be easily implemented using depth sensor information. Furthermore, depth sensor information can facilitate image labeling as described in Section 4.2.

On the other hand, due to the limited depth range of the Kinect sensor, objects have to be located between 0.4 and 9 meters from the device. Also, the depth image quality is too low in challenging outdoor environments such as in sharp sunlight. However, the results show that it is possible to use the Kinect sensor outdoors in non-direct sunlight and for cloudy days.

A continuation of this project is to improve the accuracy by adding corner and edge features that in general could describe the image structure more specifically. In later stages of this project, other types of objects like branches, leaves and peduncles will be added to the list of object classes. In addition, we may integrate laser scanner and a high resolution $R G B$ camera to improve depth and color information. Addressing the problem of over-fitting, which may occur in any learning method, we will integrate an algorithm to diagnose over-fitting and address it by a feature selection algorithm. By selecting the best subsets of features, the accuracy of the overall system may increase (see e.g. (Marcano-Cede ando et al., 2010)). Finally, we plan to use compression methods like PCA and GPLVM to maximize the overall accuracy similar to what is described in (Zhong et al., 2008).

Table 6: Confusion matrix for WMV classifier in Table 5.

\begin{tabular}{|l|l|l|l|l|l|l|}
\hline Class & Tree & Bush & Stone & Human & Total & Miss-classified \\
\hline Tree & 38 & 1 & 0 & 0 & 39 & $2 \%$ \\
\hline Bush & 0 & 58 & 0 & 0 & 58 & $0 \%$ \\
\hline Stone & 2 & 0 & 10 & 0 & 12 & $16 \%$ \\
\hline Human & 3 & 0 & 0 & 46 & 49 & $6 \%$ \\
\hline
\end{tabular}

\section{ACKNOWLEDGEMENTS}

This work was funded by the European Commission in the 7th Framework Programme (CROPS GA no 246252).

\section{REFERENCES}

An, K. H. and Park, S. H. and Chung, Y. S. and Moon, K. $Y$. and Chung, M. J. (2009). Learning discriminative 
multi-scale and multi-position Ibp features for face detection based on ada-Ida. In Robotics and Biomimetics (ROBIO), 2009 IEEE International Conference on, pages $1117-1122$.

Ästrand, B. and Baerveldt, A.-J. (2002). An agricultural mobile robot with vision-based perception for mechanical weed control. Autonomous Robots, 13:2135.

Chen, W., Shi, Y., and Xuan, G. (2007). Identifying computer graphics using hsv color model and statistical moments of characteristic functions. In Multimedia and Expo, 2007 IEEE International Conference on, pages $1123-1126$.

Cucchiara, R., Grana, C., Piccardi, M., and Prati, A. (2003). Detecting moving objects, ghosts, and shadows in video streams. Pattern Analysis and Machine Inte/ligence, IEEE Transactions on, 25(10):1337 - 1342.

Davison, A. J., Reid, I. D., Molton, N. D. and Stasse, 0. (2007). Monoslam: Real-time single camera slam. IEEE Transactions on Pattern Analysis and Machine Intelligence, 29(6):1052-1067.

De Stefano, C., Della Cioppa, A., and Marcelli, A. (2002). An adaptive weighted majority vote rule for combining multiple classifiers. In Pattern Recognition, 2002. Proceedings. 16th International Conference on, volume 2, pages $192-195$ vol.2.

Fang, Y., Lin, W., Lau, C. T., and Lee, B.-S. (2011). A visual attention model combining top-down and bottomup mechanisms for salient object detection. In Acoustics, Speech and Signal Processing (ICASSP), 2011 IEEE International Conference on, pages 1293-1296.

Friedman, N., Geiger, D. and Goldszmidt, M. (1997). Bayesian network classifiers. Machine Learning, 29(2-3):131-163.

He, L., Wang, H., and Zhang, H. (2011). Object detection by parts using appearance, structural and shape features. In Mechatronics and Automation (ICMA), 2011 International Conference on, pages 489-494.

Jain, A. K., Duin, R. P. W., and Mao, J. (2000). Statistical pattern recognition: A review. IEEE Transactions on Pattern Analysis and Machine Intelligence, 22(1):437.

James, G. and Stanford University. Dept. of Statistics (1998). Majority vote classifiers: theory and applications. Stanford University.

Jimenez-Sanchez, A., Mendiola-Santibanez, J., TerolVillalobos, I., Herrera-Ruiz, G., Vargas-Vazquez, D., Garcia-Escalante, J., and Lara-Guevara, A. (2009). Morphological background detection and enhancement of images with poor lighting. Image Processing, IEEE Transactions on, 18(3):613 -623.

Kokkinos, I., Maragos, P., and Yuille, A. (2006). Bottom-up amp; top-down object detection using primal sketch features and graphical models. In Computer Vision and Pattern Recognition, 2006 IEEE Computer Society Conference on, volume 2, pages 1893 - 1900.

Lakshmi, J. and Punithavalli, M. (2009). A survey on skeletons in digital image processing. In Digital Image Processing, 2009 International Conference on, pages 260 -269 .
Li, R., Lu, J., Zhang, Y., Lu, Z., and Xu, W. (2009). A framework of large-scale and real-time image annotation system. In Artificial Intelligence, 2009. JCAI '09. International Joint Conference on, pages 576-579.

Marcano-Cede ando, A., Quintanilla-Domi andnguez, J., Cortina-Januchs, M., and Andina, D. (2010). Feature selection using sequential forward selection and classification applying artificial metaplasticity neural network. In IECON 2010 - 36th Annual Conference on IEEE Industrial Electronics Society, pages 2845 2850.

Pordel, M., Khalilzad, N., Yekeh, F., and Asplund, L. (2011). A component based architecture to improve testability, targeted fpga-based vision systems. In Communication Software and Networks (ICCSN), 2011 IEEE 3rd International Conference on, pages $601-605$

Robert S. Lynch Jr. and Peter K. Willett (2003). Use of bayesian data reduction for the fusion of legacy classifiers. Information Fusion, 4(1):23-34.

Van den Bergh, M., Carton, D., De Nijs, R., Mitsou, N., Landsiedel, C., Kuehnlenz, K., Wollherr, D., Van Gool, L., and Buss, M. (2011). Real-time 3d hand gesture interaction with a robot for understanding directions from humans. In RO-MAN, 2011 IEEE, pages $357-362$.

Van den Bergh, M. and Van Gool, L. (2011). Combining rgb and tof cameras for real-time $3 \mathrm{~d}$ hand gesture interaction. In Applications of Computer Vision (WACV), 2011 IEEE Workshop on, pages 66 -72.

Wang, J., Wu, Q., Deng, H., and Yan, Q. (2008). Realtime speech/music classification with a hierarchical oblique decision tree. In Acoustics, Speech and Signal Processing, 2008. ICASSP 2008. IEEE International Conference on, pages $2033-2036$.

Xiao, Q., Hu, X., Gao, S., and Wang, H. (2010). Object detection based on contour learning and template matching. In Intelligent Control and Automation (WC/CA), 2010 8th World Congress on, pages 6361 -6365.

Zhang, J., Marszalek, M., Lazebnik, S., and Schmid, C. (2007). Local features and kernels for classification of texture and object categories: A comprehensive study. International Journal of Computer Vision, 73(2):213238.

Zhang, H., Berg, A. C., Maire, M. and Malik, J. (2006). Svm-knn: Discriminative nearest neighbor classification for visual category recognition. volume 2 , pages 2126-2136.

Zhong, F., Capson, D., and Schuurman, D. (2008). Parallel architecture for pca image feature detection using fpga. In Electrical and Computer Engineering, 2008. CCECE 2008. Canadian Conference on, pages $001341-001344$. 